\title{
Non-Robust Dynamic Inferences from Macroeconometric Models: Bifurcation Stratification of Confidence Regions
}

\author{
William A. Barnett* and Evgeniya Aleksandrovna Duzhak** \\ * Department of Economics, University of Kansas, Snow Hall, Lawrence, KS 66045-7585, USA. E-mail: \\ barnett@ku.edu.**Department of Economics and Finance, Baruch College, Zicklin School of Business, One Bernard \\ Baruch Way, NY, NY 10010, USA \\ November 27, 2007
}

\begin{abstract}
Grandmont (1985) found that the parameter space of the most classical dynamic models are stratified into an infinite number of subsets supporting an infinite number of different kinds of dynamics, from monotonic stability at one extreme to chaos at the other extreme, and with many forms of multiperiodic dynamics between. The econometric implications of Grandmont's findings are particularly important, if bifurcation boundaries cross the confidence regions surrounding parameter estimates in policy-relevant models. Stratification of a confidence region into bifurcated subsets seriously damages robustness of dynamical inferences.

Recently, interest in policy in some circles has moved to New Keynesian models. As a result, in this paper we explore bifurcation within the class of New Keynesian models. We develop the econometric theory needed to locate bifurcation boundaries in log-linearized New-Keynesian models with Taylor policy rules or inflation-targeting policy rules. Central results needed in this research are our theorems on the existence and location of Hopf bifurcation boundaries in each of the cases that we consider. .
\end{abstract}

Keywords: Bifurcation, Hopf bifurcation, Euler equations, New Keynesian macroeconometrics, Bergstrom-Wymer model.

\section{Introduction}

Grandmont (1985) found that the parameter space of even the simplest, classical models are stratified into bifurcation regions. This result changed prior views that different kinds of economic dynamics can only be produced by different kinds of structures. But he provided that result with a model in which all policies are Ricardian equivalent, no frictions exist, employment is always full, competition is perfect, and all solutions are Pareto optimal. Hence he was not able to reach conclusions about the policy relevance of his dramatic discovery. The econometric implications of Grandmont's findings are particularly important, if bifurcation boundaries cross the confidence regions surrounding parameter estimates in policy-relevant models. Stratification of a confidence region into bifurcated subsets damages robustness of dynamical inferences.

Grandmont was not able to reach conclusions about the policy relevance of his dramatic discovery. As a result, Barnett and He $(1999,2001,2002)$ investigated a Keynesian structural model and found results supporting Grandmont's conclusions within the parameter space of the Bergstrom-Wymer continuous-time dynamic macroeconometric model of the UK economy [see, e.g., Bergstrom, Nowmann, and Wandasiewicz (1994) regarding that model]. Criticism of Keynesian structural models by the Lucas critique have motivated development of Euler equations models having policy-invariant deep parameters, which are invariant to policy rule changes. Hence, Barnett and He $(2004,2006)$ chose to continue the investigation of policyrelevant bifurcation by searching the parameter space of the best known of the Euler equations macroeconometric models: the Leeper and Sims (1994) model. The results further confirm Grandmont's views.

Recently, interest in some policy circles has moved away from Euler equations models to New Keynesian models. In this paper we explore bifurcation within the class of New Keynesian models. In a future paper, we shall report on our results solving numerically for the location and properties of the bifurcation boundaries and their dependency upon policy-rule parameter settings. A central result used in this research is our proof of the propositions needed to establish the existence and location of Hopf bifurcation in the particular models that we consider. We find 
that a common setting of a parameter in the future-looking New-Keynesian model can put the model directly onto a Hopf bifurcation boundary.

Beginning with Grandmont's findings with a classical model, we continue to follow the path from the Bergstrom-Wymer policy-relevant Keynesian model, then to the Euler equation macroeconometric models, and now to New Keynesian models. At this stage of our research, we believe that Grandmont's conclusions appear to hold for all categories of dynamic macroeconomic models, from the oldest to the newest. ${ }^{1}$ So far, our finding suggest that Barnett and He's initial findings with the policy-relevant Bergstrom-Wymer model appear to be generic.

\section{Model}

Our analysis is centered on the New Keynesian functional structure described in this section. The main assumption of New Keynesian economic theory is that there are nominal price rigidities preventing prices from adjusting immediately and thereby creating disequilibrium unemployment. Price stickiness is often introduced in the manner proposed by Calvo (1983). The model below, used as the theoretical background for our log linearized bifurcation analysis, is based closely upon Walsh (2003), section 5.4.1, pp. $232-239$.

The demand side of the economy is modeled as an expectational, forward-looking IS curve:

$$
x_{t}=E_{t} x_{t+1}-\left(\frac{1}{\sigma}\right)\left(i_{t}-E_{t} \pi_{t+1}\right)+u_{t},
$$

where, $\pi_{\mathrm{t}}$ is the inflation rate at time $\mathrm{t} ; i_{t}$ is the nominal interest rate; $x_{t}=\left(\hat{y}_{t}-\hat{y}_{t}^{f}\right)$ is the gap between actual output percentage deviation from steady-state output, $\hat{y}_{t}$, and the flexible-price output percentage deviation from steady-state output, $\hat{y}_{t}^{f}$; and $u_{t} \equiv E_{t} \hat{y}_{t+1}^{f}-\hat{y}_{t}^{f}$. The degree of relative risk aversion is $\sigma$.

The supply side of the economy is represented by equation

$$
\pi_{t}=\beta E_{t} \pi_{t+1}+\kappa x_{t},
$$

where $\beta$ is the discount factor.

The remaining necessary equation will be a monetary policy rule, in which the central bank uses a nominal interest rate as the policy instrument. We initially center our analysis on the following specification of the current-looking Taylor rule:

$$
i_{t}=a_{1} \pi_{t}+a_{2} x_{t},
$$

where $a_{1}$ is the coefficient of the central bank's reaction to inflation and $a_{2}$ is the coefficient of the central bank's reaction to the output gap. We also consider the forward-looking and the hybrid Taylor rule.

Among targeting rules, the recent literature proposes many definitions of an inflation target. $^{2}$ We consider inflation targeting policies of the form:

$$
i_{t}=a_{1} \pi_{t},
$$

which is a current-looking inflation targeting rule. Forward-looking inflation targeting will also be considered.

When we use the current-looking Taylor rule, we are left with these three equations.

\footnotetext{
${ }^{1}$ Over the past three decades, an enormous literature has evolved on endogenous business cycles from complex dynamics; but we focus our discussion on Grandmont's early contribution, since it was the surprising nature of his results and the subsequent controversies about policy relevance that motivated our line of research.

${ }^{2}$ See Bernanke et al. (1999), Svensson (1999), and Gavin (2003).
} 


$$
\begin{aligned}
& x_{t}=E_{t} x_{t+1}-\frac{1}{\sigma}\left(i_{t}-E_{t} \pi_{t+1}\right), \\
& \pi_{t}=\beta E_{t} \pi_{t+1}+\kappa x_{t}, \\
& i_{t}=a_{1} \pi_{t}+a_{2} x_{t} .
\end{aligned}
$$

This 3-equation system constitutes a New Keynesian model, which can be written in the form $\mathbf{A} E_{\mathrm{t}} \mathbf{x}_{\mathrm{t}+1}=\mathbf{B} \mathbf{x}_{\mathrm{t}}$, where $\mathbf{x}_{\mathrm{t}}$ is the state vector, and $\mathbf{A}$ and $\mathbf{B}$ are matrices of parameters ${ }^{3}$

\section{Bifurcation Analysis}

We study the dynamic solution behavior of an $n$-dimensional state vector, $\mathbf{x}$, as the parameter vector, $\alpha$, varies. Dynamic systems undergo a bifurcation, if the parameters pass through a critical (bifurcation) point, defined as follows.

Definition 3.1: Appearance of a topologically nonequivalent phase portrait under variation of parameters is called a bifurcation.

At the bifurcation point the structure may change its stability, split into new structures, or merge with other structures. We look at local bifurcations within small neighborhoods of a fixed point, $\mathbf{x}^{*}=\mathbf{f}\left(\mathbf{x}^{*}, \boldsymbol{\alpha}\right)$, to conduct local bifurcation analysis.

The bifurcations of a map can be characterized by the eigenvalues of the Jacobian of the first derivatives of the map, computed at the bifurcation point. Let $\mathbf{J}=\mathbf{f}(\mathbf{x}, \boldsymbol{\alpha})_{\mathbf{x}}$ be the Jacobian matrix. The eigenvalues, $\lambda_{1}, \lambda_{2}, \ldots, \lambda_{n}$, of the Jacobian are also referred to as multipliers. Bifurcation will occur, if there are eigenvalues of $\mathbf{J}$ on the unit circle that violate the hyperbolicity condition. Non-hyperbolic equilibria are not structurally stable and hence generically lead to bifurcations as a parameter is varied.

In the special case of $n=2$, the following well known theorem is based upon the Hopf Bifurcation Theorem in Gandolfo (1996, ch. 25, p. 492).

Theorem 3.1: (Existence of Hopf Bifurcation in 2 Dimensions) Consider a map $\mathbf{x} \mapsto \mathbf{f}(\mathbf{x}, \boldsymbol{\alpha})$, where $\mathbf{x}$ has 2 dimensions. For each $\alpha$ in the relevant region, suppose that there is a continuously differentiable family of equilibrium points, $\mathbf{x}^{*}=\mathbf{x}^{*}(\alpha)$, at which the eigenvalues of the Jacobian are complex conjugates, $\lambda_{1}=\theta(\mathbf{x}, \boldsymbol{\alpha})+i \omega(\mathbf{x}, \boldsymbol{\alpha})$ and $\lambda_{2}=\theta(\mathbf{x}, \boldsymbol{\alpha})-i \omega(\mathbf{x}, \boldsymbol{\alpha})$. Suppose that for one of those equilibria, $\left(\mathbf{x}^{*}, \alpha^{*}\right)$, there is a critical value $\alpha_{c}$ for one of the parameters, $\alpha_{i}^{*}$, in $\alpha^{*}$ such that:

(a) The modulus of the eigenvalues becomes unity at $\alpha=\alpha^{*}$, but the eigenvalues are not roots of unity. Formally, $\lambda_{1}, \lambda_{2} \neq 1$ and $\bmod \left(\lambda_{1}\right)=\bmod \left(\lambda_{2}\right)=+\sqrt{\theta^{2}+\omega^{2}}=1$.

$$
\left.\frac{\partial\left|\lambda_{j}\left(\mathbf{x}^{*}, \boldsymbol{\alpha}^{*}\right)\right|}{\partial \alpha_{i}^{*}}\right|_{\alpha_{i}^{*}=\alpha_{c}} \neq 0 \text { for } \mathrm{j}=1,2 .
$$

Then there is an invariant closed curve Hopf bifurcation at $\alpha^{*}$.

\footnotetext{
${ }^{3}$ Detailed description of the model and these results are provided in Barnett and Duzhak (2006).

${ }^{4}$ Note that we use the notations mod $\left(\lambda_{\mathrm{j}}\right)$ and $\left|\lambda_{\mathrm{j}}\right|$ interchangeably to designate modulus of a complex variable.
} 
This theorem only applies with a $2 \times 2$ Jacobian. The more general case requires the rest of the eigenvalues to have a real part less than zero.

\subsection{Current-Looking Taylor Rule}

The matrix $\mathbf{J}$ is the Jacobian of the New Keynesian model presented above:

$$
\mathbf{J}=\left[\begin{array}{cc}
1+\frac{a_{2} \beta+k}{\sigma \beta} & \frac{a_{1} \beta-1}{\sigma \beta} \\
-\frac{k}{\beta} & \frac{1}{\beta}
\end{array}\right] .
$$

We apply Theorem 3.1 to the Jacobian of the log-linearized New Keynesian model, $\mathbf{A E}_{\mathrm{t}} \mathbf{x}_{\mathrm{t}+1}=\mathbf{B} \mathbf{x}_{\mathrm{t}}$. The characteristic equation of the Jacobian is:

$$
\begin{array}{ll} 
& \lambda^{2}-b \lambda+c=0, \\
\text { where } b=\left[1+\frac{a_{2} \beta+k}{\sigma \beta}+\frac{1}{\beta}\right] \text { and } & c=\left[\frac{\sigma \beta+a_{2} \beta+k a_{1} \beta}{\sigma \beta^{2}}\right] .
\end{array}
$$

In order to acquire a pair of complex conjugate eigenvalues, the discriminant $D$ must be strictly negative:

$$
D=b^{2}-4 c=\left[1+\frac{a_{2} \beta+k}{\sigma \beta}+\frac{1}{\beta}\right]^{2}-4 \frac{\sigma \beta+a_{2} \beta+k a_{1} \beta}{\sigma \beta^{2}}<0 .
$$

Given the sign of the parameters, the discriminant could be either positive or negative. We assume that the discriminant is negative, so that the roots of the characteristic polynomial are complex conjugate: $\lambda_{1}=\theta+i \omega$ and $\lambda_{2}=\theta-i \omega$, where $\theta=b / 2$ is the real part, $i \omega$ is the imaginary part, and $\omega=\sqrt{D} / 2=(1 / 2) \sqrt{b^{2}-4 c}$.

We choose a bifurcation parameter to vary, with other parameters constant. The model is parameterized by $\boldsymbol{\alpha}=\left[\begin{array}{lllll}\beta & \sigma & \kappa & a_{1} & a_{2}\end{array}\right]$. Relevant candidates for a bifurcation parameter are coefficients for the monetary policy rule, $a_{1}$ and $a_{2}$, about which we prove the following.

Proposition 3.1: The new Keynesian model with current-looking Taylor rule, (2.1), (2.2), and (2.3), undergoes a Hopf bifurcation, if and only if the discriminant of the characteristic equation is negative and $a_{2}^{c}=\sigma \beta-\kappa a_{1}-\sigma$.

Proof: Assume that the system, (2.1), (2.2), (2.3), produces a Hopf bifurcation. By definition, Hopf bifurcation is characterized by the appearance of a pair of complex conjugate multipliers that lie on the unit circle. Since the multipliers are complex conjugate, the discriminant has to be strictly negative.

By condition (a) for Hopf bifurcation, $\bmod \left(\lambda_{1}\right)=\bmod \left(\lambda_{2}\right)=+\sqrt{\theta^{2}+\omega^{2}}=1$. Substituting $\theta=b / 2$ and $\omega=\sqrt{D} / 2$ into that equation, we get

$$
\sqrt{\frac{1}{2}\left(1+\frac{a_{2} \beta+\kappa}{\sigma \beta}+\frac{1}{\beta}\right)+\frac{1}{4}\left[\frac{4\left(\sigma \beta+a_{2} \beta+k a_{1} \beta\right)}{\sigma \beta^{2}}-\left(1+\frac{a_{2} \beta+k+\sigma}{\sigma \beta}\right)^{2}\right]}=1 .
$$

After solving for $a_{2}$, we find that the critical value for the parameter is $a_{2}^{c}=\sigma \beta-\kappa a_{1}-\sigma$. 
Conversely, assume that the discriminant, $D$, is negative and that $a_{2}=\sigma \beta-\kappa a_{1}-\sigma$. Substituting for $a_{2}$ into the left hand side of equation (i), we find immediately that $\bmod \left(\lambda_{1}\right)=\bmod \left(\lambda_{2}\right)=1$, thereby satisfying condition (a) for Hopf bifurcation.

It can be shown as follows that the derivative of the modulus with respect to $a_{2}$ is a nonzero expression:

$$
\left.\frac{\partial\left|\lambda_{1}\right|}{\partial a_{2}}\right|_{a_{2}=\alpha_{2}^{c}}=\left.\frac{\partial\left|\lambda_{2}\right|}{\partial a_{2}}\right|_{a_{2}=\alpha_{2}^{c}}=\left.\frac{1}{2 \sigma \beta}\left(\frac{\beta \sigma}{\sigma+a_{2}+\kappa a_{1}}\right)^{\frac{1}{2}}\right|_{a_{2}=\alpha_{2}^{c}}=\frac{1}{2 \sigma \beta} \neq 0
$$

which is condition (b) for Hopf bifurcation. Hence, both conditions of the Hopf bifurcation theorem are satisfied.

\subsection{Forward-looking Taylor Rule}

A forward-looking Taylor rule sets the interest rate according to expected future inflation rate and output gap, in accordance with the following equation:

The resulting Jacobian has the form

$$
i_{t}=a_{1} E_{t} \pi_{t+1}+a_{2} E_{t} x_{t+1} \text {. }
$$

$$
\mathbf{J}=\left[\begin{array}{cc}
\frac{\sigma}{\left(\sigma-a_{2}\right)}+\frac{\kappa\left(1-a_{1}\right)}{\left(\sigma-a_{2}\right) \beta} & \frac{\left(a_{1}-1\right)}{\left(\sigma-a_{2}\right) \beta} \\
-\frac{\kappa}{\beta} & \frac{1}{\beta}
\end{array}\right] .
$$

The characteristic equation is

$$
\lambda^{2}-b \lambda+c=0
$$

where $b=\frac{\sigma \beta+\kappa\left(1-a_{1}\right)+\left(\sigma-a_{2}\right)}{\left(\sigma-a_{2}\right) \beta}$ and $c=\operatorname{det}(\mathbf{J})$.

To acquire complex conjugate eigenvalues, the discriminant $D$ must be strictly negative:

$$
D=\left(\frac{\sigma \beta+\sigma-a_{2}}{\beta\left(\sigma-a_{2}\right)}+\frac{\kappa \sigma\left(1+a_{1}\right)}{\left(1-a_{2}\right) \beta}\right)^{2}-\frac{4 \sigma}{\beta\left(\sigma-a_{2}\right)}<0 .
$$

We assume that the discriminant is negative, so that the roots of the characteristic polynomial are complex conjugate: $\lambda_{1}=\theta+i \omega$ and $\lambda_{2}=\theta-i \omega$, where $\theta=b / 2$ is the real part, $i \omega$ is the imaginary part, and $\omega=\sqrt{D} / 2$.

We need to choose a bifurcation parameter to vary while holding other parameters constant. The model is parameterized by $\boldsymbol{\alpha}=\left[\begin{array}{lllll}\beta & \sigma & \kappa & a_{1} & a_{2}\end{array}\right]$. Candidates for a bifurcation parameter are coefficients, $a_{1}$ and $a_{2}$, for the monetary policy rule. We prove the following proposition.

Proposition 3.2: The New Keynesian model with forward-looking Taylor Rule, (2.1), (2.2), and (3.1), undergoes a Hopf bifurcation, if and only if the discriminant of the characteristic equation is negative and $a_{2}^{c}=-\frac{\sigma}{\beta}+\sigma$. 
Proof: Assume that a system consisting of (2.1), (2.2), and (3.1) produces a Hopf bifurcation. Hopf bifurcation is characterized by the appearance of the pair of complex conjugate multipliers that lie on the unit circle. Then the discriminant has to be strictly negative.

By condition (a) for Hopf bifurcation, $\bmod \left(\lambda_{1}\right)=\bmod \left(\lambda_{2}\right)=+\sqrt{\theta^{2}+\omega^{2}}=1$. Substituting $\theta=b / 2$ and $\omega=\sqrt{D} / 2$ into that equation, we get $\sqrt{\frac{1}{4}\left(\frac{\left(1-a_{2}\right)\left(\sigma \beta+\sigma-a_{2}\right)+\kappa \sigma\left(1+a_{1}\right)\left(\sigma-a_{2}\right)}{\left(\sigma-a_{2}\right)\left(1-a_{2}\right) \beta}\right)^{2}+\frac{1}{4}\left(4 \frac{\sigma}{\beta\left(\sigma-a_{2}\right)}-\left(\frac{\sigma}{\sigma-a_{2}}+\frac{1}{\beta}+\frac{\kappa \sigma\left(1+a_{1}\right)}{\left(1-a_{2}\right) \beta}\right)^{2}\right)}=1$.

Solving for $a_{2}$, we find that the critical value for the parameter is $a_{2}^{c}=-\frac{\sigma}{\beta}+\sigma$.

Conversely assume that the discriminant of characteristic equation (3.2) is negative and that $a_{2}^{c}=-\frac{\sigma}{\beta}+\sigma$. Hopf bifurcation will arise if there is a pair of complex conjugate roots of (3.2) and if conditions (a) and (b) of Theorem 3.1 are satisfied.

Since the discriminant has a negative sign, roots of (3.2) have to be complex conjugate. Condition (a) of Theorem 3.1 states that for a Hopf bifurcation to arise, the modulus of the eigenvalues should be equal to unity. To show that condition (a) holds, substitute $a_{2}=-\frac{\sigma}{\beta}+\sigma$ into the left hand side of equation (ii) to find $\bmod \left(\lambda_{1}\right)=1$. Since characteristic roots are complex conjugate, it follows that $\bmod \left(\lambda_{1}\right)=\bmod \left(\lambda_{2}\right)=1$, thereby satisfying condition (a) for Hopf bifurcation

It can be shown as follows that the derivative of the modulus with respect to $a_{2}$ is a nonzero expression:

$$
\left.\frac{\partial\left|\lambda_{1}\right|}{\partial a_{2}}\right|_{a_{2}=\alpha_{2}^{c}}=\left.\frac{\partial\left|\lambda_{2}\right|}{\partial a_{2}}\right|_{a_{2}=\alpha_{2}^{c}}=\frac{\beta}{\sigma} \neq 0
$$

which is condition (b) for Hopf bifurcation. Hence, both conditions of the Hopf bifurcation theorem are satisfied.

\subsection{Hybrid Taylor Rule:}

Consider the Taylor rule of the following form:

$$
i_{t}=a_{1} E_{t} \pi_{t+1}+a_{2} x_{t}
$$

where the interest rate is set according to forward-looking inflation and current-looking output gap. A rule of that form was proposed in Clarida, Gali and Gertler (1999). This form of the rule is intended to capture the central bank's existing policy. Substituting equation (3.3) into the consumption Euler equation we acquire the Jacobian,

$$
\mathbf{J}=\left[\begin{array}{cc}
1+\frac{a_{2}}{\sigma}+\frac{\kappa\left(1-a_{1}\right)}{\beta \sigma} & -\frac{1-a_{1}}{\sigma \beta} \\
-\frac{\kappa}{\beta} & \frac{1}{\beta}
\end{array}\right],
$$

with the associated characteristic polynomial 


$$
\lambda^{2}-b \lambda+c=0
$$

where $b=\frac{1}{\beta}+1+\frac{\beta a_{2}-\kappa\left(a_{1}-1\right)}{\sigma \beta}$ and $c=\operatorname{det}(\mathbf{J})=\frac{1}{\beta}+\frac{a_{2}}{\sigma \beta}$.

To get complex conjugate eigenvalues, the discriminant $D$ must be strictly negative:

$$
D=\left(\frac{\sigma(1+\beta)+\beta a_{2}-\kappa\left(a_{1}-1\right)}{\sigma \beta}\right)^{2}-\frac{4\left(\sigma+a_{2}\right)}{\sigma \beta}<0 .
$$

We assume that the discriminant is negative, so that the roots of the characteristic polynomial are complex conjugate: $\lambda_{1}=\theta+i \omega$ and $\lambda_{2}=\theta-i \omega$, where $\theta=b / 2$ is the real part, $i \omega$ is the imaginary part, and $\omega=\sqrt{D} / 2$.

We choose a bifurcation parameter to vary while holding other parameters constant. Coefficients for the monetary policy rule, $a_{1}$ and $a_{2}$ are candidates for a bifurcation parameter.

Proposition 3.3: The new Keynesian model with Hybrid-Taylor rule, equations (2.1), (2.2), (3.3), undergoes a Hopf bifurcation, if and only if the discriminant of the characteristic polynomial (3.4) is negative and $a_{2}^{c}=\beta \sigma-\sigma$.

Proof: Assume that a system consisting of (2.1), (2.2), and (3.3) produces a Hopf bifurcation. Then there exists a pair of complex conjugate multipliers that lie on the unit circle. Since the multipliers are complex conjugate, the discriminant is negative.

By condition (a) for Hopf bifurcation, $\bmod \left(\lambda_{1}\right)=\bmod \left(\lambda_{2}\right)=+\sqrt{\theta^{2}+\omega^{2}}=1$. Substituting $\theta=b / 2$ and $\omega=\sqrt{D} / 2$ into that equation, we get

$$
\sqrt{\frac{1}{4}\left(\frac{\sigma+\beta \sigma+\kappa+\beta a_{2}-\kappa a_{1}}{\sigma \beta}\right)^{2}+\frac{1}{4}\left(4 \frac{\sigma \beta+a_{2}}{\beta \sigma}-\left(1+\frac{1}{\beta}+\frac{a_{2} \beta+\kappa\left(1-a_{1}\right)}{\sigma \beta}\right)^{2}\right)}=1 .
$$

Solving for $a_{2}$, we find that the critical value for the parameter is $a_{2}^{c}=\beta \sigma-\sigma$.

Conversely assume that the discriminant of the characteristic polynomial (3.4) is negative and $a_{2}^{c}=\beta \sigma-\sigma$. Hopf bifurcation will arise if there is a pair of complex conjugate roots of (3.4) and if conditions (a) and (b) of Theorem 3.1 are satisfied.

Since the discriminant has a negative sign, roots of (3.4) have to be complex conjugate. Condition (a) of Theorem 3.1 states that for a Hopf bifurcation to arise, the modulus of the eigenvalues should be equal to unity. Substitute $a_{2}=\beta \sigma-\sigma$ into the left hand side of equation (iii) to find $\bmod \left(\lambda_{1}\right)=1$. Since characteristic roots are complex conjugate, it follows that mod $\left(\lambda_{1}\right)=\bmod \left(\lambda_{2}\right)=1$, thereby satisfying condition (a) for Hopf bifurcation.

It can be shown as follows that the derivative of the modulus with respect to $a_{2}$ is a nonzero expression:

$$
\left.\frac{\partial\left|\lambda_{1}\right|}{\partial a_{2}}\right|_{a_{2}=\alpha_{2}^{c}}=\left.\frac{\partial\left|\lambda_{2}\right|}{\partial a_{2}}\right|_{a_{2}=\alpha_{2}^{c}}=\frac{1}{2 \beta \sigma} \neq 0
$$

which is condition (b) for Hopf bifurcation. Both conditions for Hopf bifurcation are satisfied. 


\subsection{Current-Looking Inflation Targeting}

As the third equation for New Keynesian model, we now use the inflation targeting equation

$$
i_{t}=a_{1} \pi_{t}
$$

instead of the Taylor rule. Then the Jacobian is

$$
\mathbf{J}=\left[\begin{array}{cc}
\frac{\sigma \beta+k}{\sigma \beta} & \frac{a_{1}}{\sigma}-\frac{1}{\sigma \beta} \\
-\frac{k}{\beta} & \frac{1}{\beta}
\end{array}\right]
$$

with characteristic equation

$$
\lambda^{2}-b \lambda+c=0
$$

where $\quad b=1+\frac{\sigma+\kappa}{\beta \sigma}$ and $c=\frac{1}{\beta}\left(\frac{\beta \sigma+\kappa}{\sigma \beta}\right)+\frac{\kappa}{\sigma \beta}\left(a_{1}-\frac{1}{\beta}\right)$.

To acquire complex conjugate eigenvalues, the discriminant $D$ must be strictly negative:

$$
D=\left(\frac{\sigma \beta+\sigma+\kappa}{\sigma \beta}\right)^{2}-\frac{4\left(\sigma \beta+\kappa a_{1} \beta\right)}{\sigma \beta^{2}}<0 .
$$

We assume that the discriminant is negative, so that the roots of the characteristic polynomial are complex conjugate: $\lambda_{1}=\theta+i \omega$ and $\lambda_{2}=\theta-i \omega$, where $\theta=b / 2$ is the real part, $i \omega$ is the imaginary part, and $\omega=\sqrt{D} / 2$.

We choose a bifurcation parameter to vary, while holding other parameters constant. The model is parameterized by $\boldsymbol{\alpha}=\left[\begin{array}{llll}\beta & \sigma & a_{1} & \kappa\end{array}\right]$. A candidate for a bifurcation parameter is the coefficient, $a_{1}$, of the monetary policy rule. We have the following proposition.

Proposition 3.4: The New Keynesian model with current-looking inflation targeting, equations, (2.1), (2.2), and (3.5), produces a Hopf bifurcation, if and only if the discriminant of the characteristic equation (3.6) is negative and $a_{1}^{c}=(\sigma \beta-\sigma) / \kappa$.

Proof: Assume that a system consisting of (2.1), (2.2), and (3.5) produces a Hopf bifurcation. Hopf bifurcation is characterized by the appearance of the pair of complex conjugate multipliers that lie on the unit circle. Them the discriminant has to be strictly negative.

By condition (a) for Hopf bifurcation, $\bmod \left(\lambda_{1}\right)=\bmod \left(\lambda_{2}\right)=+\sqrt{\theta^{2}+\omega^{2}}=1$. Substituting $\theta=b / 2$ and $\omega=\sqrt{D} / 2$ into that equation, we get

$$
\sqrt{\left(\frac{\sigma(\beta+1)+\kappa}{4 \sigma \beta}\right)^{2}+\frac{1}{4}\left(4\left(\frac{\kappa a_{1} \beta+\sigma \beta}{\sigma \beta^{2}}\right)^{2}-\left(\frac{(\beta+1)}{\beta}+\frac{\sigma+\kappa}{\sigma \beta}\right)^{2}\right)}=1 \text {. }
$$

Solving for $a_{1}$, we find that the critical value for the parameter is $a_{1}^{c}=(\sigma \beta-\sigma) / \kappa$. 
Conversely assume that the discriminant of the characteristic polynomial (3.6) is negative and $a_{1}^{c}=(\sigma \beta-\sigma) / \kappa$. Hopf bifurcation will arise if there is a pair of complex conjugate roots of (3.6) and if conditions (a) and (b) of Theorem 3.1 are satisfied.

Since the discriminant has a negative sign, roots of (3.6) have to be complex conjugate. Condition (a) of Theorem 3.1 states that for a Hopf bifurcation to arise, the modulus of the eigenvalues should be equal to unity. Substitute $a_{1}^{c}=(\sigma \beta-\sigma) / \kappa$ into the left hand side of equation (iv) to find $\bmod \left(\lambda_{1}\right)=1$. Since characteristic roots are complex conjugate, it follows that $\bmod \left(\lambda_{1}\right)=\bmod \left(\lambda_{2}\right)=1$, thereby satisfying condition (a) for Hopf bifurcation.

The derivative of the modulus with respect to $a_{1}$ is a non-zero expression:

$$
\left.\frac{\partial\left|\lambda_{1}\right|}{\partial a_{1}}\right|_{a_{1}=\alpha_{1}^{c}}=\left.\frac{\partial\left|\lambda_{2}\right|}{\partial a_{1}}\right|_{a_{1}=\alpha_{1}^{c}}=\frac{\kappa}{2 \beta \sigma} \neq 0 .
$$

which is condition (b) for Hopf bifurcation. Both conditions for Hopf bifurcation are satisfied.

\subsection{Forward-Looking Inflation Target Rule}

Using the following forward-looking inflation targeting rule,

$$
i_{t}=a_{1} E_{t} \pi_{t+1}
$$

as the third equation for New Keynesian model, (2.1), (2.2), (3.7), produces the Jacobian:

$$
\mathbf{J}=\left[\begin{array}{cc}
1-\frac{\kappa}{\beta \sigma}\left(a_{1}-1\right) & \frac{1}{\sigma \beta}\left(a_{1}-1\right) \\
-\frac{\kappa}{\beta} & \frac{1}{\beta}
\end{array}\right]
$$

with characteristic equation

$$
\lambda^{2}-b \lambda+c=0
$$

where

$$
b=\frac{1+\beta}{\beta}-\frac{\kappa}{\sigma \beta}\left(a_{1}-1\right) \text { and } c=\frac{\beta \sigma-\kappa\left(a_{1}-1\right)}{\sigma \beta^{2}}-\frac{\kappa\left(a_{1}-1\right)}{\sigma \beta^{2}} .
$$

To get complex conjugate eigenvalues, the discriminant $D$ must be strictly negative:

$$
D=\left(\frac{\sigma(\beta+1)-\kappa\left(a_{1}-1\right)}{\sigma \beta}\right)^{2}-\frac{4}{\beta}<0 .
$$

We assume that the discriminant is negative, so that the roots of the characteristic polynomial are complex conjugate: $\lambda_{1}=\theta+i \omega$ and $\lambda_{2}=\theta-i \omega$, where $\theta=b / 2$ is the real part, $i \omega$ is the imaginary part, and $\omega=\sqrt{D} / 2$.

We choose a bifurcation parameter to vary, while holding other parameters constant. The model is parameterized by $\boldsymbol{\alpha}=\left[\begin{array}{llll}\beta & \sigma & a_{1} & \kappa\end{array}\right]$. We have the following proposition about the forward-looking inflation-targeting New Keynesian model. Surprisingly this result does not require separate setting of $a_{1}$ to attain Hopf bifurcation. Under the conditions of this proposition, no freedom remains to select $a_{1}$ independently. 
Proposition 3.5: The New Keynesian model, (2.1), (2.2), (3.7), with forward-looking inflation targeting produces a Hopf bifurcation, if and only if the discriminant of the characteristic equation (3.8) is negative and $\beta^{c}=1$.

Proof: Assume that a system consisting of (2.1), (2.2), and (3.7) produces a Hopf bifurcation. Hopf bifurcation is characterized by the appearance of the pair of complex conjugate multipliers that lie on the unit circle. Then the discriminant has to be strictly negative.

By condition (a) for Hopf bifurcation, $\bmod \left(\lambda_{1}\right)=\bmod \left(\lambda_{2}\right)=+\sqrt{\theta^{2}+\omega^{2}}=1$. Substituting $\theta=b / 2$ and $\omega=\sqrt{D} / 2$ into that equation, we get

$$
\sqrt{\left(\frac{\sigma \beta-\kappa\left(a_{1}-1\right)+\sigma}{2 \beta \sigma}\right)^{2}+\left(\frac{1}{4}\right)\left(\left(1+\frac{1-\kappa\left(a_{1}-1\right)}{\sigma \beta}\right)^{2}-\frac{4}{\beta}\right)^{2}=1} .
$$

Solving for $\beta$ we find that the critical value for the parameter is $\beta^{c}=1$.

Conversely assume that the discriminant of the characteristic polynomial (3.8) is negative and $\beta^{c}=1$. Hopf bifurcation will arise, if there is a pair of complex conjugate roots of (3.8) and if conditions (a) and (b) of Theorem 3.1 are satisfied.

Since the discriminant has a negative sign, roots of (3.8) have to be complex conjugate. Condition (a) of Theorem 3.1 states that the modulus of the eigenvalues should be equal to unity. Substitute $\beta^{c}=1$ into the left hand side of equation (v) to find $\bmod \left(\lambda_{1}\right)=1$. It follows that mod $\left(\lambda_{1}\right)=\bmod \left(\lambda_{2}\right)=1$, thereby satisfying condition (a) for Hopf bifurcation.

It can be shown as follows that the derivative of the modulus with respect to $\beta$ is a nonzero expression:

$$
\left.\frac{\partial\left|\lambda_{1}\right|}{\partial \beta}\right|_{\beta=\beta^{c}}=\left.\frac{\partial\left|\lambda_{2}\right|}{\partial \beta}\right|_{\beta=\beta^{c}}=-\frac{1}{2} \neq 0,
$$

which is condition (b) for Hopf bifurcation. Both conditions for Hopf bifurcation are satisfied.

Parameter $\beta$ is the discount factor from the representative agent's optimization problem. It is also a coefficient in the Phillips curve scaling the impact of expected inflation. Some authors assume for simplicity that $\beta=1 .^{5}$ Surprisingly we find that setting can put the New Keynesian model with forward-looking inflation targeting directly on top of a Hopf bifurcation boundary. This conclusion is conditional upon the assumption that the log-linearized New Keynesian model is a good approximation to the economy and that the discriminant of the characteristic equation (3.8) is negative. In such cases, setting the discount factor $\beta$ equal to unity is not appropriate.

\section{Conclusion}

If a bifurcation boundary crosses into the confidence region of a model's parameter estimates, robustness of dynamic inferences is seriously compromised. Our ongoing bifurcation analysis of New Keynesian functional forms is detecting the possibility of Hopf bifurcation. This

\footnotetext{
${ }^{5}$ See Roberts (1995), Gali and Gertler (1999).
} 
paper provides the methodology that we have developed and are using. One surprising result from the proofs in this paper is the theoretical finding that a common setting of the parameter $\beta$ in the future-looking New-Keynesian model can put the model directly onto a Hopf bifurcation boundary.

We have been analyzing the reduced log-linearized system locally. Global study of the full nonlinear system will require different tools, which will be the subject of future research. When we find Hopf bifurcation with the linearized system, the result is sufficient but not necessary for existence of a bifurcation boundary.

In this paper, we develop the formulas and prove the propositions we are using in detecting bifurcation boundaries in the parameter spaces of New Keynesian models. Subsequent papers will provide our empirical results. Future research also will explore backward-looking monetary policy rules.

\section{References}

[1] Barnett W.A. and Duzhak E.A. "Non-Robust Dynamic Inferences from Macroeconometric Models: Bifurcation Stratification of Confidence Regions", University of Kansas Working Paper 200608, (2006).

[2] Barnett, William A. and Yijun He, "Stability Analysis of Continuous-Time Macroeconometric Systems," Studies in Nonlinear Dynamics and Econometrics, January, vol 3, no. 4, (1999), 169-188.

[3] Barnett, William A. and Yijun He, "Nonlinearity, Chaos, and Bifurcation: A Competition and an Experiment," with Yijun He, in Takashi Negishi, Rama Ramachandran, and Kazuo Mino (eds.), Economic Theory, Dynamics and Markets: Essays in Honor of Ryuzo Sato, Kluwer Academic Publishers, (2001), 167-187.

[4] Barnett, William A. and Yijun He, "Stabilization Policy as Bifurcation Selection: Would Stabilization Policy Work if the Economy Really Were Unstable?," Macroeconomic Dynamics, vol 6, no 5, November, (2002), 713747.

[5] Barnett, William A. and Yijun He, "Bifurcations in Macroeconomic Models," in Steve Dowrick, Rohan Pitchford, and Steven Turnovsky (eds), Economic Growth and Macroeconomic Dynamics: Recent Developments in Economic Theory, Cambridge University Press, (2004), 95-112.

[6] Barnett, William A. and Yijun He, "Robustness of Inferences to Singularity Bifurcation," with Yijun He, Proceedings of the Joint Statistical Meetings of the 2005 American Statistical Society, vol. 100, American Statistical Association, February, (2006).

[7] Bergstrom, A. R., K. B. Nowmann, and S. Wandasiewicz, "Monetary and Fiscal Policy in a Second-order Continuous Time Macroeconometric Model of the United Kingdom," Journal of Economic Dynamics and Control, 18, (1994), 731-761.

[8] Bernanke, Ben S., Thomas Laubach, Frederic S. Mishkin, and Adam S. Posen .,Inflation Targeting: Lessons from the International Experience. Princeton, NJ:Princeton University Press,(1999).

[9] Calvo, G., "Staggered Prices in a Utility-Maximizing Framework," Journal of Monetary Economics, 12, (1983), 383-398.

[10] Clarida, Richard, Jordi Galí, and Mark Gertler, "The Science of Monetary Policy: A New Keynesian Perspective,” Journal of Economic Literature 37, December, (1999), 1661-1707.

[11] Gali, J., and M. Gertler, "Inflation dynamics: a structural econometric analysis," Journal of Monetary Economics. 44, October, (1999), 195-222.

[12] Gandolfo, Giancarlo, Economic Dynamics, Third edition, New York and Heidelburg, Springer, (1996).

[13] Gavin, William T, “Inflation Targeting: Why It Works and How To Make It Work Better," Federal Reserve Bank of Saint Louis Working Paper 2003-027B, (2003).

[14] Grandmont, J. M., "On Endogenous Competitive Business Cycles," Econometrica, 53, (1985), 995-1045.

[15] Leeper, E. and C. Sims, “Toward a Modern Macro Model Usable for Policy Analysis," NBER Macroeconomics Annual, (1994), 81-117.

[16] Roberts, J. M., "New Keynesian Economics and the Phillips Curve", Journal of Money, Credit and Banking, 27(4), November, (1995), 975-984. 
[17] Shapiro, A. H., "Estimating the New Keynesian Phillips Curve: A Vertical Production Chain Approach" Federal Reserve Bank of Boston Working Paper No. 06-11, (2006).

[18] Svensson, Lars E. O., "Inflation Targeting as a Monetary Policy Rule." Journal of Monetary Economics 43, (1999), 607-54.

[19] Walsh, Carl E., Monetary Theory and Policy, 2nd edition MIT Press: Cambridge Mass, (2003). 